\title{
THE THREE COMPONENTS OF D-SPIN
}

\author{
Peter G. 0. Freund
}

The Enrico Fermi Institute and the Department of Physics

The University of Chicago, Chicago, 111 inois, 60637 


\section{DISCLAIMER}

This report was prepared as an account of work sponsored by an agency of the United States Government. Neither the United States Government nor any agency Thereof, nor any of their employees, makes any warranty, express or implied, or assumes any legal liability or responsibility for the accuracy, completeness, or usefulness of any information, apparatus, product, or process disclosed, or represents that its use would not infringe privately owned rights. Reference herein to any specific commercial product, process, or service by trade name, trademark, manufacturer, or otherwise does not necessarily constitute or imply its endorsement, recommendation, or favoring by the United States Government or any agency thereof. The views and opinions of authors expressed herein do not necessarily state or reflect those of the United States Government or any agency thereof. 


\section{DISCLAIMER}

Portions of this document may be illegible in electronic image products. Images are produced from the best available original document. 


\title{
THE THREE COMPONENTS OF D-SPIN *
}

\author{
Peter G. 0. Freund \\ The Enrico Fermi Institute \\ and the Department of Physics
}

The University of Chicago, Chicago, I1linois, 60637

\begin{abstract}
The concept of D-spin, previously introduced to account for the selection rules for exotic hadron couplings, is further investigated. The three components of D-spin are identified. $D_{3}$ turns out to be a simple topological characteristic of the way a hadron couples at a vertex. $D_{3}$ is conserved at hadronic vertices. In hadronic N-point functions an "effective $\mathrm{D}_{3}$ " is conserved. It is shown that in the presence of three species of quarks $(\mathcal{H}, \lambda, \lambda)$ there are three D-spins. An application of the D-spin formalism to diffractive exotic hadron production is presented.
\end{abstract}

* Supported in part by the U. S. Atomic Energy Commission. 


\section{INTRODUCTION}

No hadrons have ever been observed to decay into two protons, into two positive pions, into two K-mesons, etc.... This was originally interpreted as evidence against the existence of exotic hadrons,i.e. of hadrons with total quark number (number of quarks plus number of antiquarks) $N>3$. Using the dual ${ }^{1)}$ two component (background and resonance) picture $^{2)}$ of hadronic reactions, it was shown ${ }^{3)}$ that exotic hadrons of arbitrarily large ${ }^{4} \mathrm{~N}$ ought to exist. The absence of resonances from channels like pp, $\pi^{+} \pi^{+}, \mathrm{K}^{+} \mathrm{K}^{+}$, etc.... is then accounted for, by a selection rule. ${ }^{5)}$ It was suggested that this selection rule can be interpreted as the conservation of a new spin-like quantity: the D-spin. ${ }^{6)}$ For this interpretation to hold up, one has to identify the components $D_{1}$, $D_{2}$ and $D_{3}$ of $D$-spin and see what one means by, say, a baryon of $D=1 / 2$, $D_{3}=-1 / 2$. In this paper we shall identify the components of D-spin and show that $D_{3}$ is indeed a new additive quantum number at hadronic vertices. We shall also find that at hadronic vertices $D_{3}$ and $\vec{D}^{2}$ are conserved but not $D_{1}$ and $D_{2}$. In hadronic $N$-point functions $D_{3}$ conservation can be violated as propagators do not conserve $D_{3}$. However, even for $\mathrm{N}$-point functions, a conserved additive quantum number the "effective $\mathrm{D}_{3}$ " or $\bar{D}_{3}$ can be defined.

We then show that for the important case of three species of quarks $\mathcal{S}, \mathcal{N}, \lambda$ one can define three types of $D$-spin.

Finally we apply the D-spin formalism to the problem of exotic hadron production. 
2. REVIEW OF D-SPIN *

To start our discussion let us disregard baryon number, hypercharge and isospin and simply label a hadron by its total quark number N. Thus a nonexotic (i.e. qa-type) meson becomes $\mathrm{H}_{2}$, a baryon (qqq) $\mathrm{H}_{3}$, etc...From fig. la we see that in $\mathrm{H}_{2} \mathrm{H}_{2}$ scattering, $\mathrm{H}_{2}$ Regge-exchange builds up $\mathrm{H}_{2}$ resonances in the direct channel. On the other hand in $\mathrm{H}_{3} \mathrm{H}_{3}$ scattering, the same Regge exchange builds up direct channel exotic $\mathrm{H}_{4}$ resonances (fig. 1b). 7) From $\mathrm{H}_{3} \mathrm{H}_{4}$ scattering we then see, that $\mathrm{H}_{2}$ exchange builds up $\mathrm{H}_{5}$ resonances in the direct channel (fig. 1c). Continuing this procedure we see that $\mathrm{H}_{2}$ exchange builds up through duality exotic hadrons $H_{N}$ with arbitrarily high total quark number $\mathrm{N}$. Now in a process like $\mathrm{H}_{2} \mathrm{H}_{2}$ scattering we were able to insure duality without $\mathrm{H}_{4}$ intermediate states or $\mathrm{H}_{4}$ exchanges. Nevertheless from $\mathrm{H}_{3} \mathrm{H}_{3}$ scattering we were led to the existence of $\mathrm{H}_{4}$ mesons. In order not to upset the carefully achieved duality-exchange degeneracy pattern in $\mathrm{H}_{2} \mathrm{H}_{2}$ scattering, it is required that the coupling $\mathrm{H}_{4} \mathrm{H}_{2} \mathrm{H}_{2}$ be forbidden 3 ). Similarly the couplings $\mathrm{H}_{6} \mathrm{H}_{3} \mathrm{H}_{3}, \mathrm{H}_{6} \mathrm{H}_{4} \mathrm{H}_{2}$ etc....... have to be forbidden. One readily convinces oneself that all these couplings are indeed forbidden and a consistent duality-exchange degeneracy pattern emerges if at any allowed hadronic vertex ${ }^{5)}$ :

A) each of the three hadrons meeting at the vertex, exchanges at least one quark line with each of the remaining two hadrons and

\footnotetext{
* The reader familiar with the work of references 5) and 6) may omit this section.
} 
B) no quark line is connected with an antiquark line from the same hadron.

The principles $A$ ) and $B$ ) then lead without much further ado to the selection rule ${ }^{5)}$;

S) for the coupling $A B C$ to be allowed it is necessary that the following three inequalities hold $\left(N_{A}=\right.$ total quark number of $A$, etc...)

$$
\begin{aligned}
& N_{A} \leqslant N_{B}+N_{C}-2 \\
& N_{B} \leqslant N_{C}+N_{A}-2 \\
& N_{C} \leqslant N_{A}+N_{B}-2
\end{aligned}
$$

Defining the D-spin of a hadron ${ }^{6)}$ by

$$
D=\frac{N}{2}-1,
$$

the relations (1) are equivalent to

$$
\left|D_{B}-D_{C}\right| \leqslant D_{N} \leqslant D_{B}+D_{C}
$$

Baryon conservation and the definition (2) then require the differences $D_{B}+D_{C}-D_{A}$ and $D_{B}+P_{C}-\left|D_{B}-D_{C}\right|$ to be integers. Thus $D_{A}$ can take only the values $\left|D_{B}-D_{C}\right|,\left|D_{B}-D_{C}\right|+1, \ldots, D_{B}+D_{C}$. At this point the identification of $D$ with a spin-like quantity becomes highly suggestive. The selection rule $S$ then states that at hadronic vertices $D$-spin is conserved. To complete the identification of $D$ with a spin-like quantity we have to find the individual components of D-spin. This will be done in the next section. 
Here we still make some useful observations.

i) D-spin conservation holds not only at hadronic vertices but for $N$-point functions in the tree-approximation. Diagrams with loops break D-spin conservation. This is illustrated in fig. 2 where a $D=1$ hadron $\mathrm{H}_{4}$ decays into two nonexotic $(D=0)$ mesons via a loop diagram. As in a dual theory resonances are narrow, diagrams with loops (i.e. final state interactions with the exception of Pomeranchuk type diagrams) are expected to be small and consequently D-spin conservation is expected to hold rather accurately. As we shall see later, the small branching ratio $\phi \rightarrow \rho \pi / \phi \rightarrow K \bar{K}$ is a measure of D-spin breaking.

ii) Inclusion of baryon number and of other additive quantum numbers would not change our discussion of this section. There is only one point to keep in mind, the proliferation of exotica discussed above,leads to arbitrarily high values of all these quantum numbers. Thus, for instance there must exist $H_{6}$ with baryon number $B=0$ $\left(q^{3-3}\right)$ but also $H_{6}$ with $B=2\left(q^{6}\right)$. Similarly $H_{9}$ with $B=1,3$ and so on. That this is so can be seen from fig. 3 . We now give a few applications of D-spin conservation. First, a meson $\mathrm{H}_{4}$ has $\mathrm{D}=1$ so that it cannot decay into two nonexotic $(D=0)$ mesons but can decay in a baryon-antibaryon $\left(\mathrm{H}_{3} \mathrm{H}_{3}\right)$ pair as for $\mathrm{H}_{3}$ $D=1 / 2$. This explains why the channels $\pi^{+} \pi^{+}, \mathrm{K}^{+} \mathrm{K}^{+}$are empty of resonances. As a further example,the D-spin of the proton being $1 / 2$, a. pp state can only couple to a $D=0$ or $a D=1$ hadron. Baryon conservation, on the other hand requires that the baryon number of this hadron equal two, whence its $D \geqslant 2$. So $D$-spin and baryon conservation 
together forbid the appearance of resonances in the pp-system. of course, through final state interactions, small resonant effects may be induced even in the pp system (see remark $i$ above).

\section{IDENTIFICATION OF THE THREE COMPONENTS OF D-SPIN}

If the interpretation of $D$ as a spin is to make sense, then we have to understand what we mean by $D_{3}$ (the third component of D-spin). Consider three hadrons $A, B$ and $C$ such that the $A B C$ vertex be allowed. To this vertex we attach a sense of rotation say clockwise $A \rightarrow B \rightarrow$ $C \rightarrow A\left(\right.$ fig. 4). The total quark number of $A$ is $N_{A}$. of these $N_{A}$ quark lines $N_{A Q}$ go clockwise (i.e. to the hadron $B$ ) and $N_{A Q}$ counterclockwise (i.e. to the hadron $C$ ). Obviously (because of rule $B$ section 2)

$$
N_{A Q}+N_{A C}=N_{A}=2 D_{A}+1
$$

Define

$$
\left(D_{3}\right)_{A}=\frac{1}{2}\left(N_{A Q}-N_{A Q N}\right)
$$

and similar definitions for $\left(D_{3}\right)_{B}$ and $\left(D_{3}\right)_{C}$. It is then clear that

$$
\left(D_{3}\right)_{A}+\left(D_{3}\right)_{B}+\left(D_{3}\right)_{C}=0
$$

so that $D_{3}$ is conserved at the $A B C$ vertex. Because of rule $A$ section 2, for any hadron 


$$
N_{Q} \geqslant 1, N_{Q} \geqslant 1
$$

so that from (3.1) and (3.2) we see that the possible values of $D_{3}$ are

$$
-D,-D+1, \ldots \ldots \ldots, D
$$

These are precisely the possible values of the third component of a spin whose value is $D$. The additive quantity

$$
D_{3}=\frac{1}{2}\left(N_{Q}-N_{O}\right.
$$

is thus conserved and has the obvious interpretation of third component of D-spin. As simple examples, we present in fig. 5 the various $D_{3}$ states of $\mathrm{H}_{2}, \mathrm{H}_{3}, \mathrm{H}_{4}$. From the above, it is obvious that the $\mathrm{D}_{3}$ state of a hadron $A$ in the $A B C$ vertex depends on the cyclic order of the hadrons $A, B, C$ at the vertex.

$$
\text { Now, } D_{1} \text { and } D_{2} \text { or more conveniently, } D_{ \pm}=D_{1} \pm i D_{2} \text {, are easily }
$$

identified. As $D_{+}\left(D_{-}\right)$has to be a raising (lowering) operator, it must lead from a state $\left|D, D_{3}\right\rangle$ to a state $\mid D, D_{3}+1>\left(\mid D, D_{3}-1>\right)$, so that it has to flip a counterclockwise (clockwise) quark line into a clockwise (counterclockwise) line. So, now we have identified $D_{ \pm}, D_{3}$ and $\vec{D}^{2}$. We also know that $D_{3}$ and $\vec{D}^{2}$ are conserved. How about 
$D_{+}$and $D_{-}$? To answer this question, all one has to consicier is the $\mathrm{H}_{4} \mathrm{H}_{4} \mathrm{H}_{2}$ vertex. If all three components of $\mathrm{D}$-spin were conserved then the $\mathrm{H}_{2}$ (with $\mathrm{D}=0$ ) ought to couple with equal strengths to an $\mathrm{H}_{4}$ $(D=1)$ with $D_{3}$ value of +1 , 0 or -1 . From fig. 6 however, we see that for the $\mathrm{H}_{4} \mathrm{H}_{4} \mathrm{H}_{2}$. coupling to be possible, the $\mathrm{D}_{3}$ value of $\mathrm{H}_{4}$ must be +1 or -1 . It is impossible to couple $\mathrm{H}_{2}$ to an $\mathrm{H}_{4}$ in the $\mathrm{D}_{3}=0$ state. This proves that $D_{1}$ and $D_{2}$ cannot be conserved. Thus $D_{3}$ and $\vec{D}^{2}$ are conserved at a hadronic vertex but not $D_{1}$ and $D_{2}-$ This is not hard to picture. For instance, if the interaction hamiltonian depended on $\mathrm{D}_{3}$, then it would commute with $\mathrm{D}_{3}$ and $\overrightarrow{\mathrm{D}}^{2}$ but not with $\mathrm{D}_{1}$ or $\mathrm{D}_{2}$. We now come to the problem of $\mathrm{D}_{3}$ - conservation in hadronic $\mathrm{N}$ point functions. This will be discussed in the next section.

\section{EFFECTIVE $D_{3}$ VALUES AND N-POINT FUNCTIONS}

$D_{3}$ being conserved at hadronic vertices, one would naively think that it must be conserved in hadronic $N$-point functions as well. After all, the latter are built out of vertices. Unfortunately this is not quite so. The point is that the vertices, making up an N-point function, are connected by "propagators" and these do not necessarily conserve $D_{3}$. As a simple example consider the quark diagram of fig. 7 . Here the s-channel $\mathrm{H}_{4}$ intermediate state couples with $\mathrm{D}_{3}=0$ to the baryons on the left but with $\mathrm{D}_{3}=+1$ to the $\mathrm{H}_{4} \mathrm{H}_{2}$ on the right. Thus while propagating, its $D_{3}$ has changed from 0 to +1 . Does this mean that we cannot speak of $D_{3}$ for hadronic $N$-point functions? No. To see how we can define $D_{3}$ for $N$-point functions we shall consider the special case 
of 4-point functions. We divide the quark diagrams for 4-point functions in three categories:

I Planar diagrams. By a planar diagram we mean a quark diagram for which the four external hadrons can be labeled 1, 2, 3, 4 in such a way that every quark line that "enters" the diagram at the hadron $i$ "exits" either at the hadron $i+1$ or $i-1(\bmod 4)$. An example of a planar diagram is given in fig. 8.

II Quasi planar diagrams. These are all quark diagrams which are not planar in the sense of I, but can be drawn in a plane without any lines crossing. For an example see Fig. 9a.

II Nonplanar diagrams. These are all quark diagrams which cannot be drawn in a plane without lines crossing. An example is given in figures 10a - 10c which show really the same diagram once as an stonce as a tu- and once as an su-diagram. As a rule nonplanar diagrams have poles in all three ( $s, t$ and $u$ ) channels. Such diagrams were previously considered in reference 6 (under the name of $s-t-u$ diagrams). It was observed there that in a dual resonance model, such diagrams could be described by Virasoro ${ }^{8)}$, rather than Veneziano ${ }^{9)}$ type terms. The conformal theory of such Virasoro terms has been recently developed ${ }^{10)}$. This solves the ambiguity, as to the treatment of such "s-t-u" diagrams, mentioned in ref. 6 . Nonplanar 4-point diagrams can be drawn without crossing lines on a sphere.

With this classification of diagrams we are now in measure to discuss $D_{3}$-conservation for the 4-point function.

It is obvious that $D_{3}$ is exactly conserved in planar diagrams. 
In the case of quasiplanar and nonplanar diagrams, it is not in general possible, to attach a given $D_{3}$ value to each of the external particles. Take diagram 9a for instance. The baryon $C$ has $D_{3}=-1 / 2$ in the $\mathrm{CDH}_{3 \mathrm{~s}}$ but $\mathrm{D}_{3}=+1 / 2$ in the $\mathrm{BCH}_{4}$ t vertex. To be able to attach a unique $\mathrm{D}_{3}$ value to each external or internal hadron one has to first isolate a "planar core" of the diagram by removing the "diagonal" lines. Fig. 9b shows the planar core of fig. 9a. For this planar core we can attach uniquely a new "effective". $D_{3}$ value $\bar{D}_{3}$ to each hadron. For instance the baryon $C$ now carries the "effective $D_{3}$ " value $\bar{D}_{3}=0$. Obviously since lines have been removed from the original diagram, it can happen, as indeed it does in the example under consideration, that $\bar{D}_{3}$ should be integer (half-integer) even though $D_{3}$ and $D$ are half integer (integer). In any case $\bar{D}_{3}$ D. It is readily seen that the effective $D_{3}$ of a hadron, as defined above, is really the average value of $D_{3}$ over all the vertices in which this hadron participates. As an example, for the baryon $C$ in fig. 9, $\bar{D}_{3}=0, D_{3 a v}=\left(+\frac{1}{2}-\frac{1}{2}\right) / 2=0$. So for quasiplanar diagrams $\bar{D}_{3}$ is conserved. Similarly, for the hadrons in intermediate states in such diagrams, $\overline{\mathrm{D}}_{3}$ is just the average value of $\mathrm{D}_{3}$ of that hadron over the two vertices (with proper relative orientation!) in which it participates. Thus for instance the baryon $\mathrm{H}_{3 \mathrm{~s}}$ as incoming to the vertex $\mathrm{H}_{3 \mathrm{~s}} \mathrm{DC}$ has $\mathrm{D}_{3}=+1 / 2$ while as outgoing from the vertex $\mathrm{ABH}_{3 \mathrm{~s}}$ has $\mathrm{D}_{3}=-1 / 2$ so that $i$ ts $\bar{D}_{3}=0=\bar{D}_{3 A}+\bar{D}_{3 B}=-\bar{D}_{3 C}-\bar{D}_{3 D}$.

Planar and quasiplanar diagrams for 4-point functions, can have poles in two channels only. Now, if we look at nonplanar diagrams, these have poles in all three channels so there are three different ways of drawing any nonplanar diagram $(s-t, t-u, u-s)$ so that it has poles in 
both "cyclic" channels appearing in the plane of the figure. We call these the three versions of the diagram. An example of the three versions of one diagram is presented in fig. 10. For each of these versions one can define a $\bar{D}_{3}$ for each particle as the average of the $D_{3}^{\prime} s$ with which the particle participates in the two vertices corresponding to the cyclic channels of that version. The so defined $\bar{D}_{3}$ is conserved just as in the quasiplanar case. However one and the same particle will have different values of $\bar{D}_{3}$ in the three versions of the diagram. This can be seen for example in figures $10 \mathrm{a}-\mathrm{c}$, where the values of $\bar{D}_{3}$ are written in. So, for a nonplanar diagram one has to indicate the $\bar{D}_{3}$ values for each of its three versions.

To sum up:

A) for planar diagrams $D_{3}$ is conserved;

B) for quasiplanar diagrams $D_{3}$ is neither uniquely defined for each hadron, nor conserved, but for each hadron there exists a unique value $\bar{D}_{3}$ (the average or effective $D_{3}$ ) which is conserved;

C) for nonplanar diagrams neither $\mathrm{D}_{3}$ nor $\overline{\mathrm{D}}_{3}$ are uniquely defined for each hadron, but for each version of that diagram, there exists a uniquely defined $\bar{D}_{3}$ for each hadron appearing in the diagram, and moreover this $\overline{\mathrm{D}}_{3}$ is conserved.

It is obvious that this procedure can be applied also to $\mathrm{N}$-point functions with $N \geqslant 5$. 


\section{THREE KINDS OF D-SPIN}

The quark model used in hadrodynamics, employs three species of quarks, $Q$, Nand $\lambda$. For each of these three species individually, a principle of the type $B$ of section 2 holds, however a principle of the type $A$ does not hold. For example, two of the hadrons meeting at one vertex could exchange no $\lambda-1$ ike quarks, but only $Q$ and/or $\mathcal{N}$ quarks. (see fig. 7). It is therefore necessary to define

$$
D^{(i)}=\frac{N}{2}^{(i)} \quad\left(I=8, X^{P}, \lambda\right)
$$

where $N(i)$ is the number of $i$ type quarks and antiquarks contained in the hadron. From here on, it will be convenient to use the notation $i=1,2,3$ for $\mathcal{Q}, \mathcal{N}, \lambda$ respectively. Then the total $D$-spin of section 2 is

$$
D=\sum_{i=1}^{3} D^{(i)}-1
$$

Similarly

$$
D_{3}^{(i)}=\frac{1}{2}(N \underset{Q}{(i)}-N(i)
$$

Here $N_{Q}^{(i)}\left(N_{Q}^{(i)}\right)$ is the number of quark and antiquark lines of type $i$ that leave the hadron clockwise (counterclockwise) at the vertex. 
Again

$$
D_{3}=\Sigma D_{3}^{(i)}
$$

We can now easily generalize the arguments' of section 3 to find that at a hadronic vertex $\vec{D}_{2}^{2}\left(\vec{D}^{(1)}\right),{ }^{2}\left(\vec{D}^{(2)}\right)^{2},\left(\vec{D}^{(3)}\right)^{2}, D_{3}, D_{3}{ }_{3}^{(1)}, D_{3}{ }_{3}^{(2)}$,

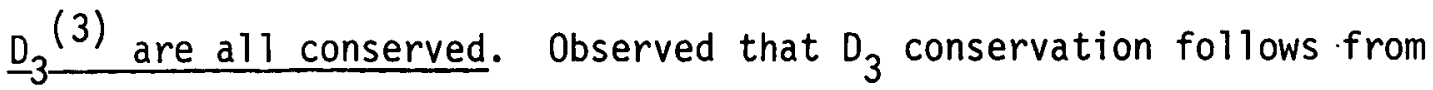
$\mathrm{D}_{3}{ }^{(i)}$ conservation and eq. (4.4), but the conservation of $\vec{D}^{2}$ does not follow from those of $\vec{D}^{(i) 2}$ (because of the extra -1 in eq. (4.2)). Thus $\vec{D}^{2}$ conservation is a separate requirement that leads to additional results.

A simple example is now in order. The decay $\phi \rightarrow \rho \pi$ is forbidden by $D^{(3)}$ conservation. Indeed $D^{(3)}=1$ for $\phi$ but $D^{(3)}=0$ for $\rho$ and $\pi$. On the other hand this same decay is also forbidden by $\mathrm{D}_{3}{ }^{(1)}, \mathrm{D}_{3}{ }^{(2)}$ and $D$ conservation. Indeed for the decay $\phi \rightarrow \rho^{+} \pi^{-}$, as can be seen from fig. 8 , for the $\rho^{+}, D_{3}{ }^{(1)}=1 / 2, D_{3}{ }^{(2)}=1 / 2$, so that $D_{3}=1 / 2+1 / 2=1$. This is impossible since for the $\rho, D=0$ and therefore, the only possible value of $D_{3}$ is zero. This example substantiates the remark made earlier that the small $\phi \rightarrow \rho \pi / \phi \rightarrow K \bar{K}$ brancing ratio is a measure of $D$-(more precisely $D^{(3)}-$ ) spin nonconservation. Effective $D_{3}{ }^{(i)}(I=1,2,3)$ for $N$-point functions can be constructed along the lines of section 4 . 
6. D-SPIN AND EXOTIC HADRON PRODUCTION

In diagrams with loops, D-spin is not conserved. Diffractive processes being unitarity effects, are presumably described by diagrams with loops. ${ }^{11)}$ As such, for diffractive processes one does not expect D-spin to be conserved. Thus diffractive processes say of the type $\underline{\pi p} \rightarrow$ Mp or $\pi p \rightarrow \pi X$ can lead to exotic $\left(D_{M} \neq 0, D_{X} \neq 1 / 2\right)$ hadron. production. $\left.{ }^{*}\right)$ One thus predicts exotic hadron production processes that have an essentially energy independent cross-section.

I wish to thank F. Mansouri and J. L. Rosner for stimulating discussions.

*) The possibility of diffractive exotic meson production has been pointed out in ref. 12. 


\section{REFERENCES}

1) R. Dolen, D. Horn and C. Schmid, Phys. Rev. 166 (1968) 1768.

2) P.G.0. Freund, Phys. Rev. Letters 20 (1968) 235; H. Harari, Phys. Rev. Letters 20 (1968) 1395.

3) J.L. Rosner, Phys. Rev. Letters 21 (1968) 950.

4) D.P. Roy and M. Suzuki, Phys. Lett. 28B (1969) 558; P.G.0. Freund and R.J. Rivers Phys. Lett. 29B (1969) 510.

5) P.G.0. Freund, R. Waltz and J.L. Rosner, Nucl. Phys. B13 (1969) 237.

6) S. Ellis, P.H. Frampton, P.G.0. Freund and D. Gordon, Nucl. Phys. B24 (1970) 465.

7) H. Harari, Phys. Rev. Letters 22 (1969) 562; J. L. Rosner, Phys. Rev. Letters 22 (1969) 689; T.Matsuoka , K. Ninomiya and S. Sawada, Progr. Theor. Phys. 42 (1969) 56.

8) M. A. Virasoro, Phys. Rev. 177 (1969) 2309.

9) G. Veneziano, Nuovo Cimento 57A (1968) 190.

10) J. Shapiro, Phys. Lett. 33B (1970) 361: see also M. Yoshimura, Berkeley preprint (1970).

11) P.G.0. Freund, Lett. al Nuovo Cimento 4 (1970) 147.

12) J. L. Rosner, University of Minnesota preprint (1970). 


\section{Figure Captions:}

Fig. 1 Quark diagrams and duality.

Fig. 2 D-spin nonconservation in one loop diagram.

Fig. 3 Duality requires both $B=2(a)$ and $B=0(b)$ hadrons $H_{6}$ of $D$-spin 2 .

Fig. 4 Definition of $\mathrm{N}_{A Q}$ and $\mathrm{N}_{A C}$

Fig. $5 D_{3}$ states of $D=0,1$ and 2 hadrons.

Fig. $6 \quad D_{1}$ and $D_{2}$ are not conserved.

Fig. $7 \quad D_{3}$-nonconservation through hadron propagation.

Fig. 8 A planar quark diagram.

Fig. 9 a) A quasi planar diagram and

b) its planar core.

Fig. 10 The three versions of a nonplanar diagram. The $\bar{D}_{3}$ assignments are obtained either by averaging $D_{3}$ over the vertices or more simply by eliminating all diagonal. lines and looking at the planar cores of the three versions. It is important to observe that the planar cores are not the same for the three versions.

Fig. 11 Vertex without a $\lambda-1$ ike quark line between two of the hadrons $\left(K^{+}\right.$and $\left.K^{-}\right)$meeting at the vertex.

Fig. $12 \mathrm{D}_{3}{ }^{(1)}, \mathrm{D}_{3}{ }^{(2)}$ and $\vec{D}^{2}$ conservation forbid $\phi \rightarrow \rho \pi$. 


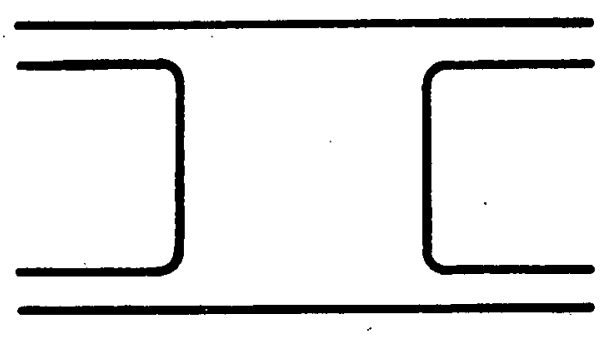

(a)

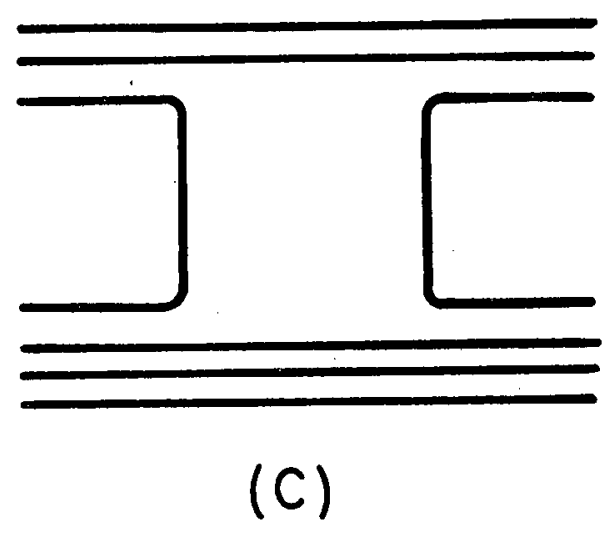

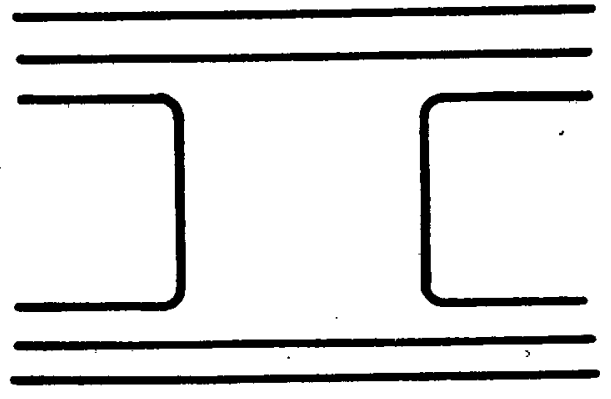

(b)

Fig. I 




Fig.2 


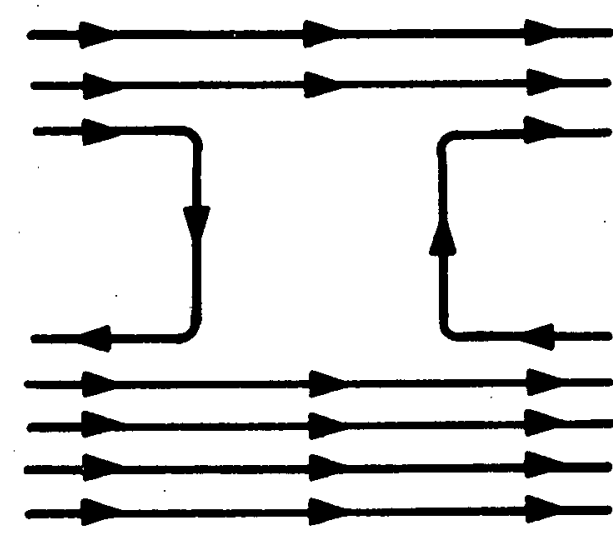

(a)

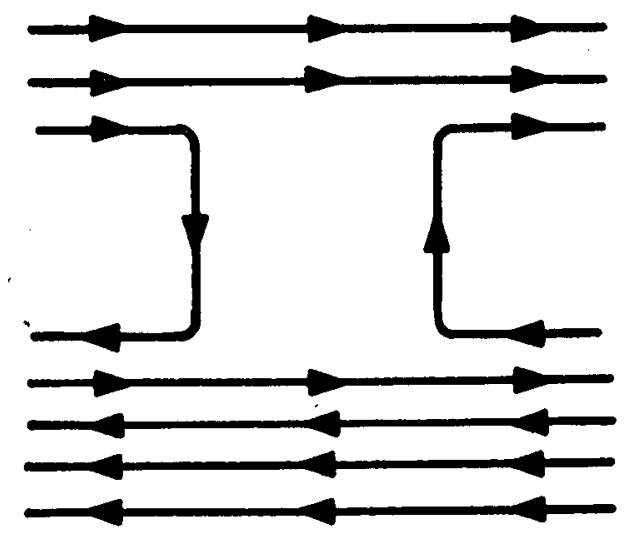

(b)

Fig.3 


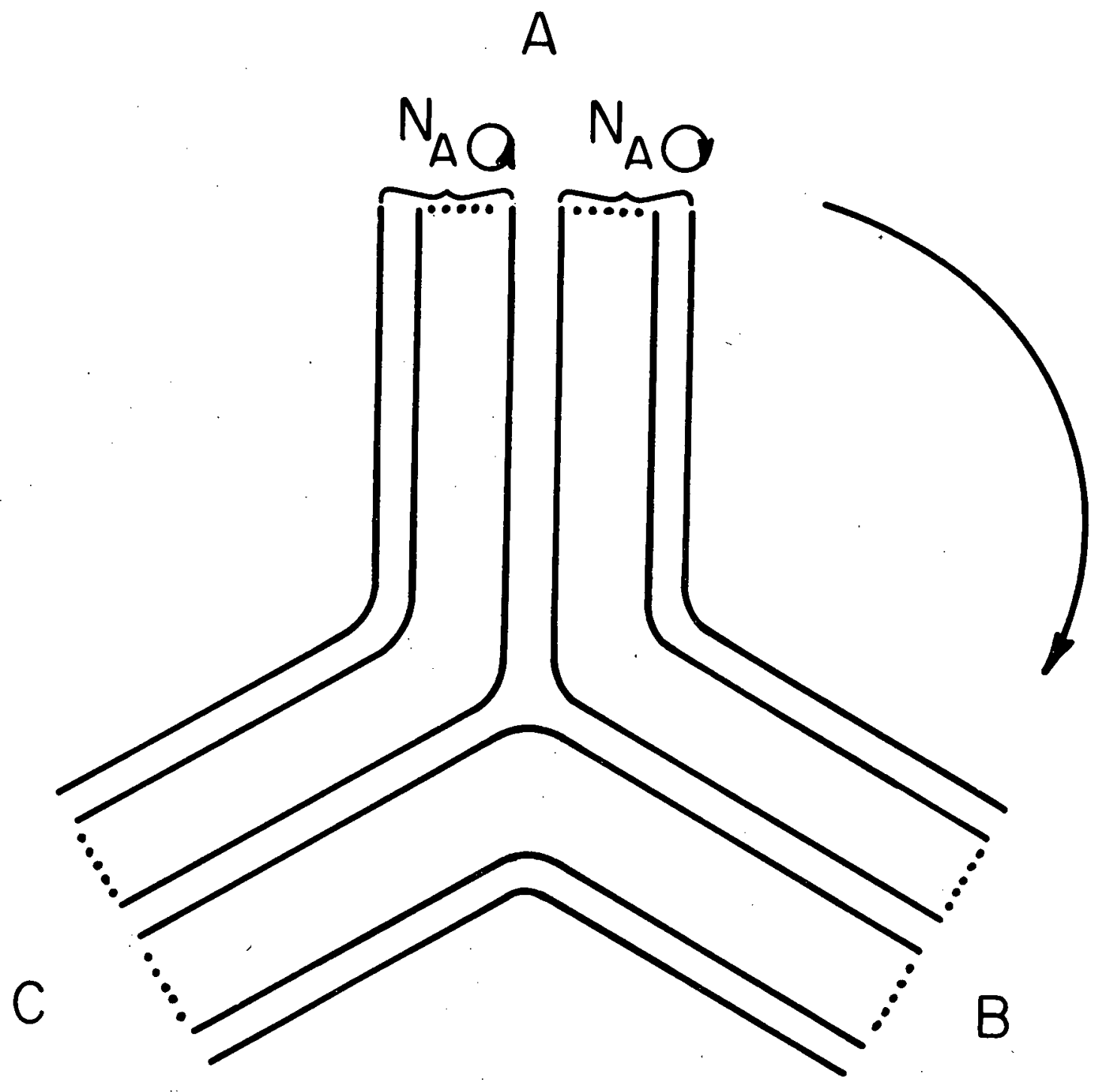

Fig. 4 

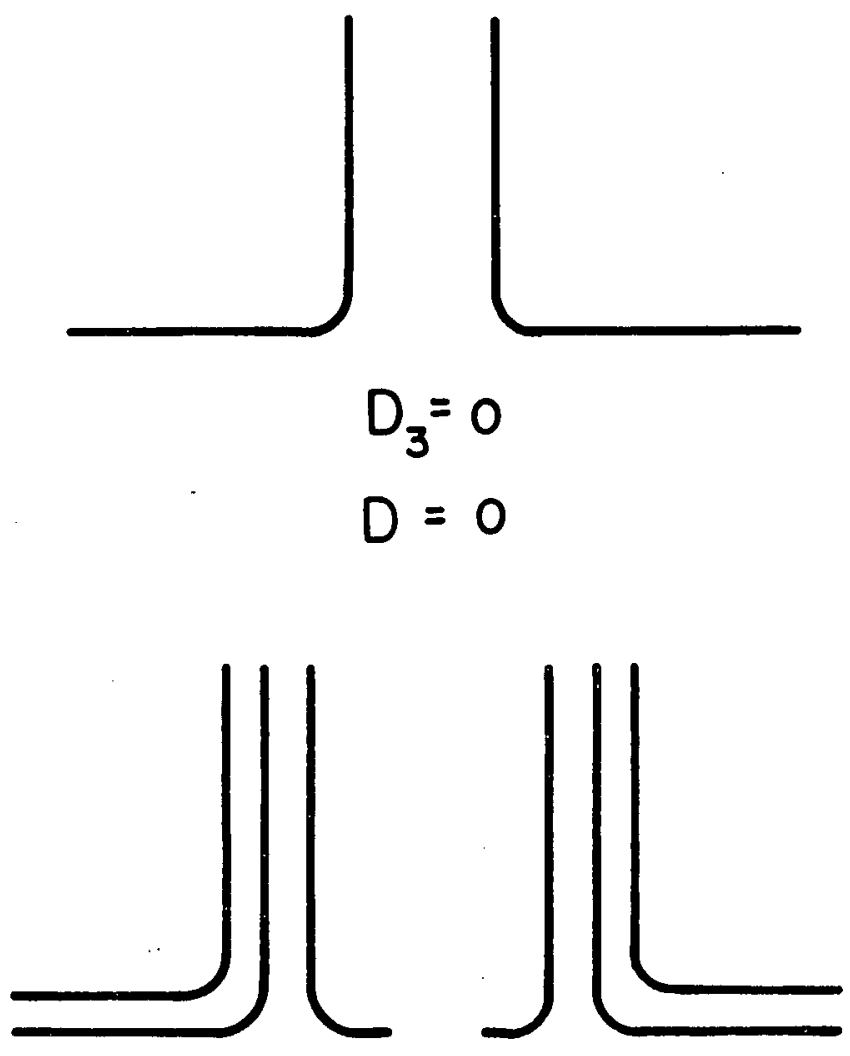

$$
D_{3}=-\frac{1}{2}
$$$$
D_{3}=+\frac{1}{2}
$$

$D=\frac{1}{2}$
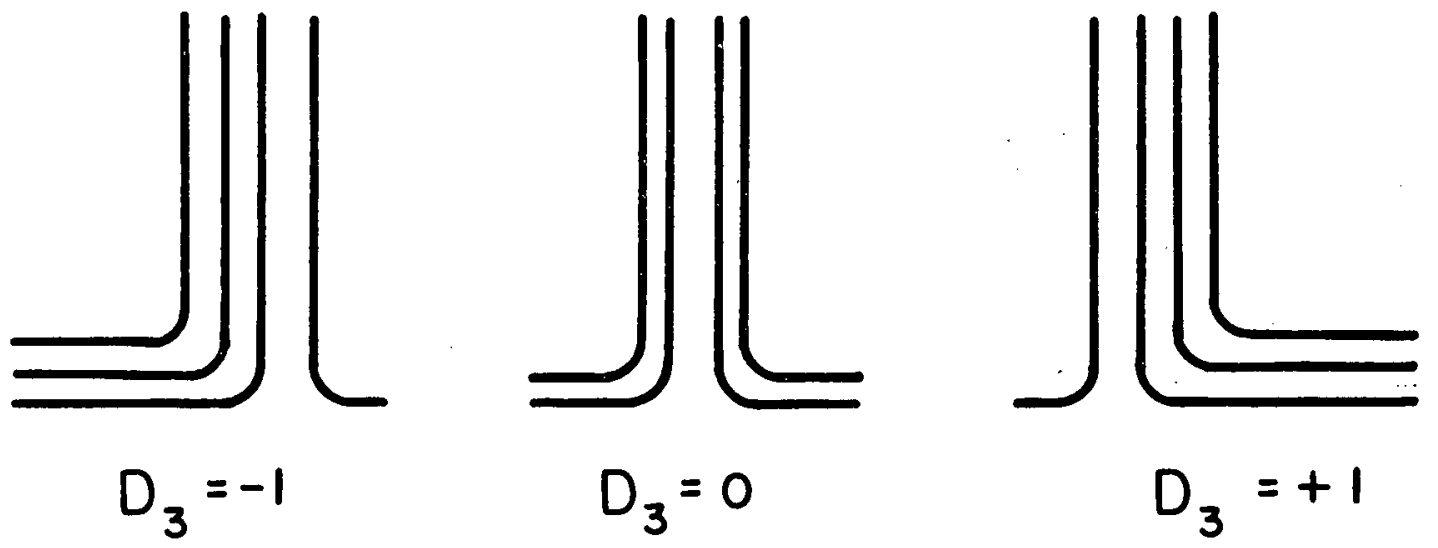

$D=1$

Fig.5 


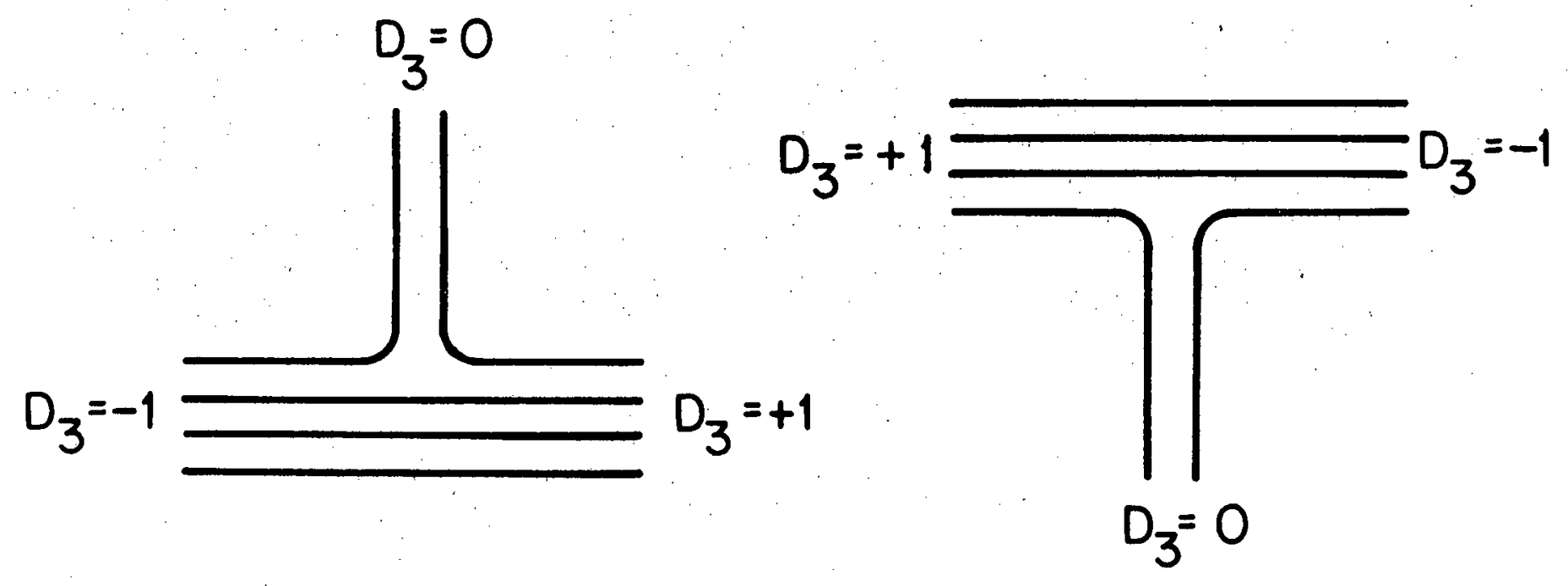

Fig.6 


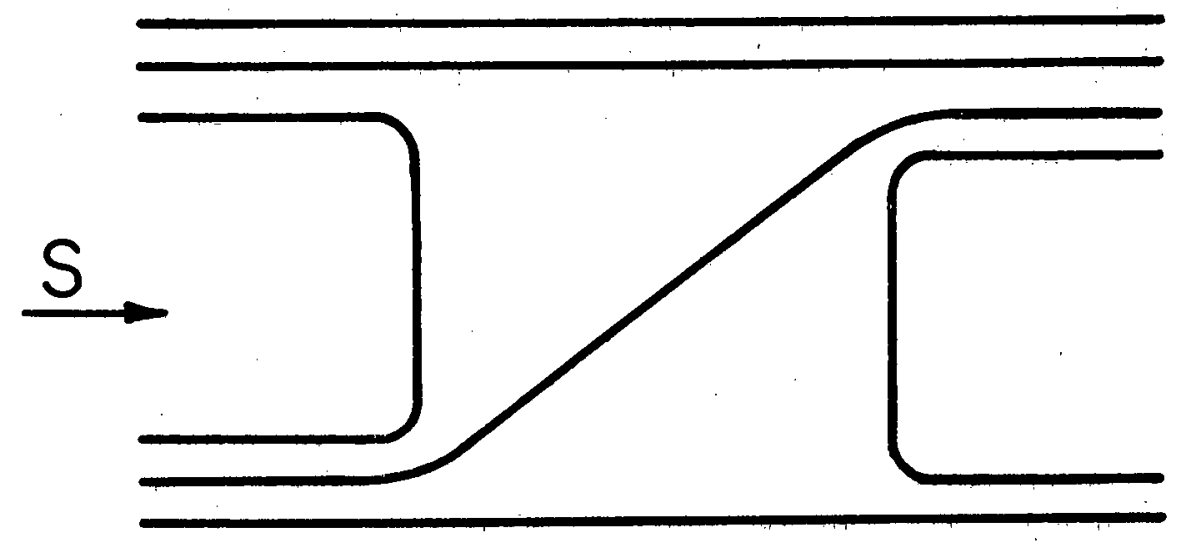

Fig.7 


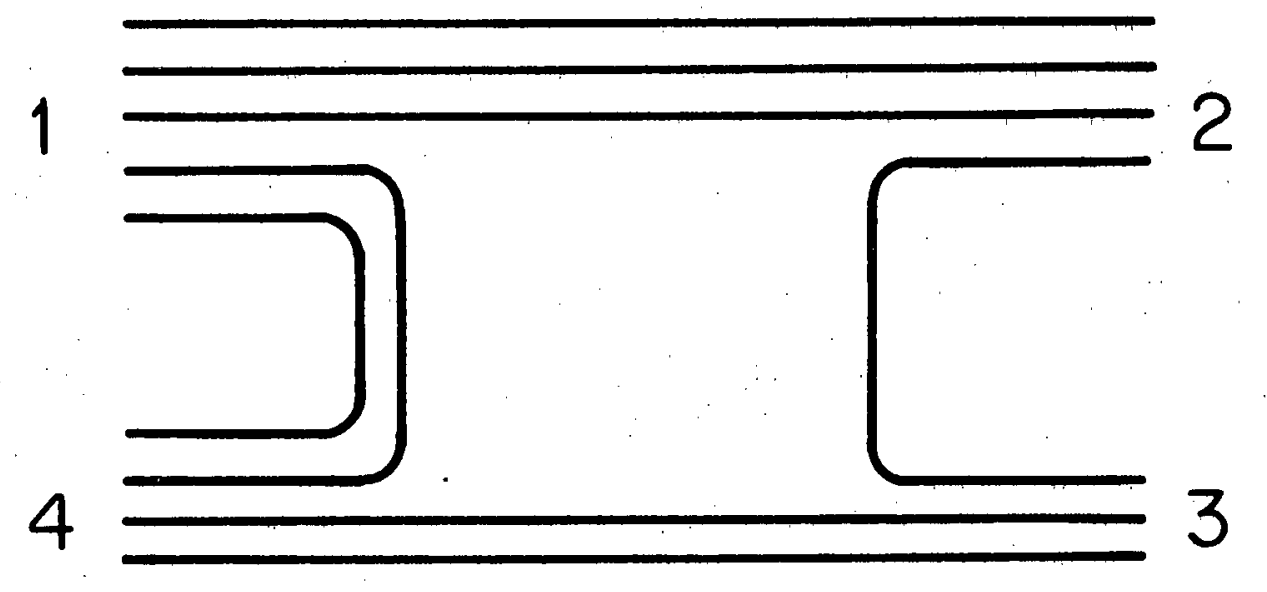

Fig.8 


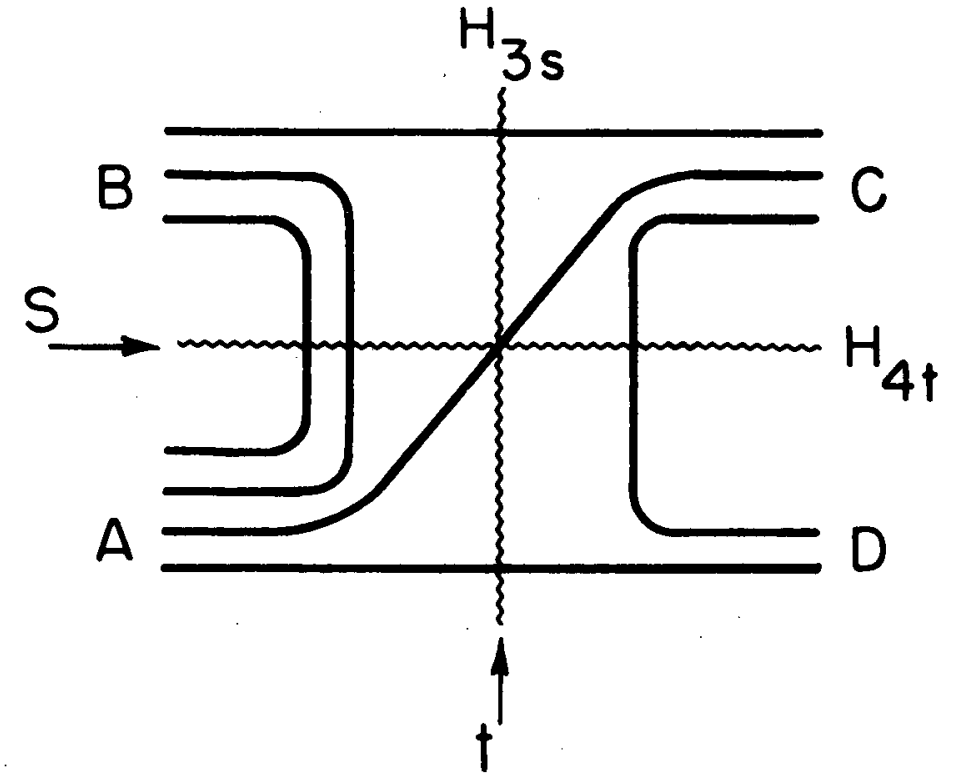

(a)

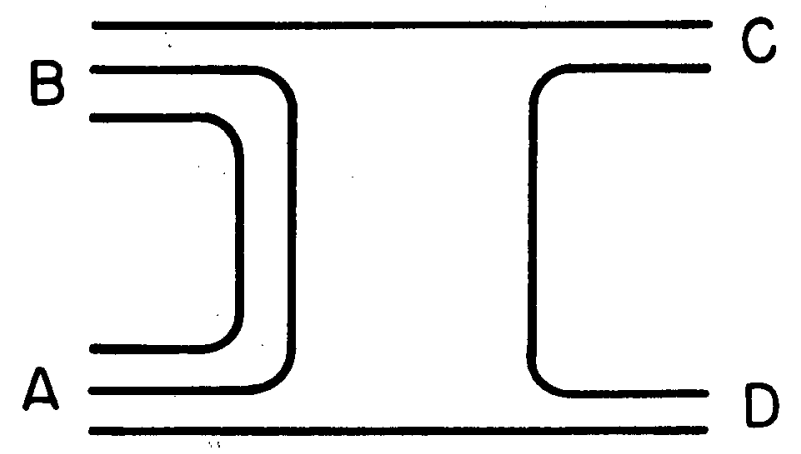

(b)

Fig. 9 


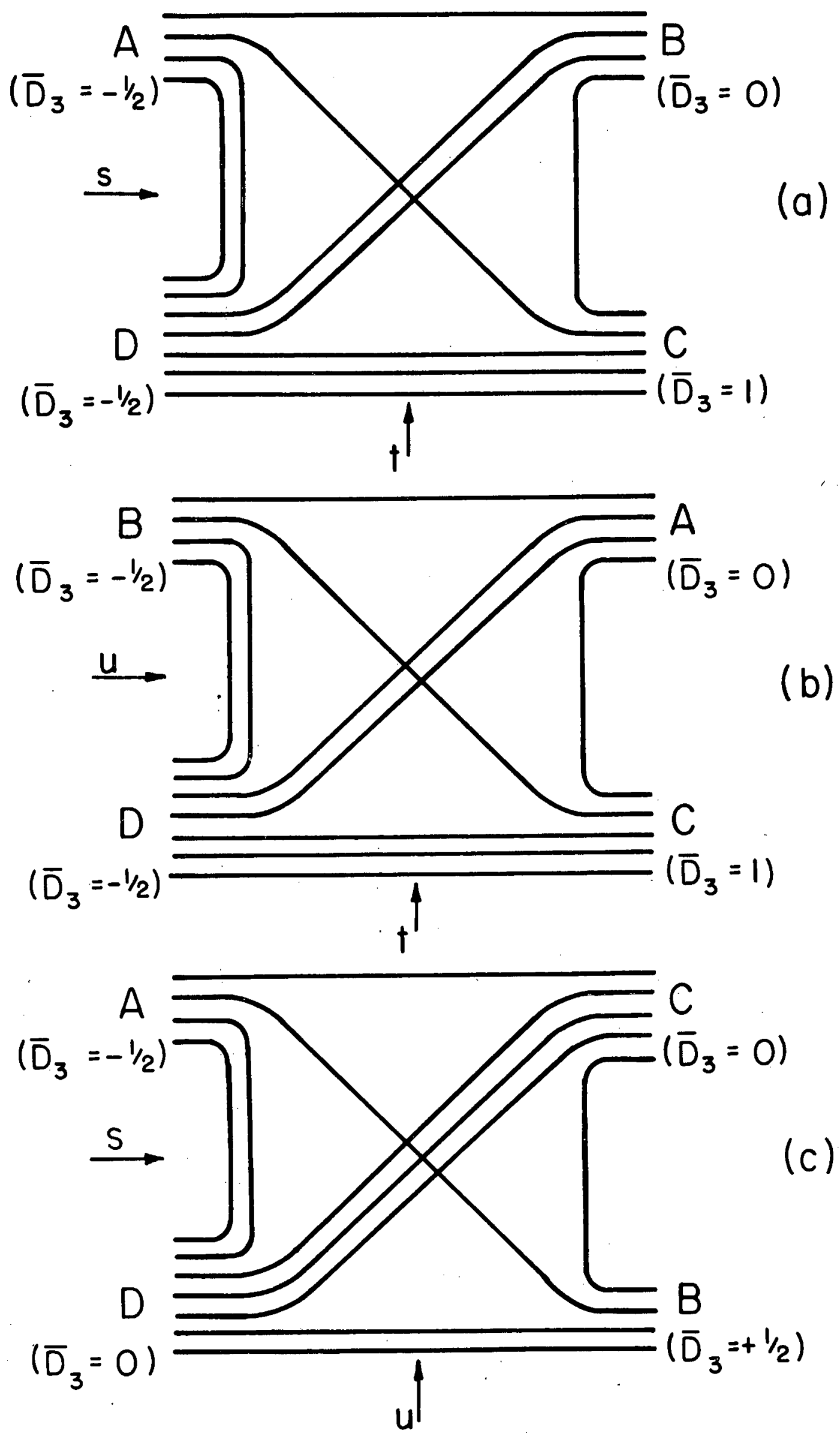

Fig. 10 
<smiles>[Y]C([Y])[3H]</smiles>

Fig.ll 


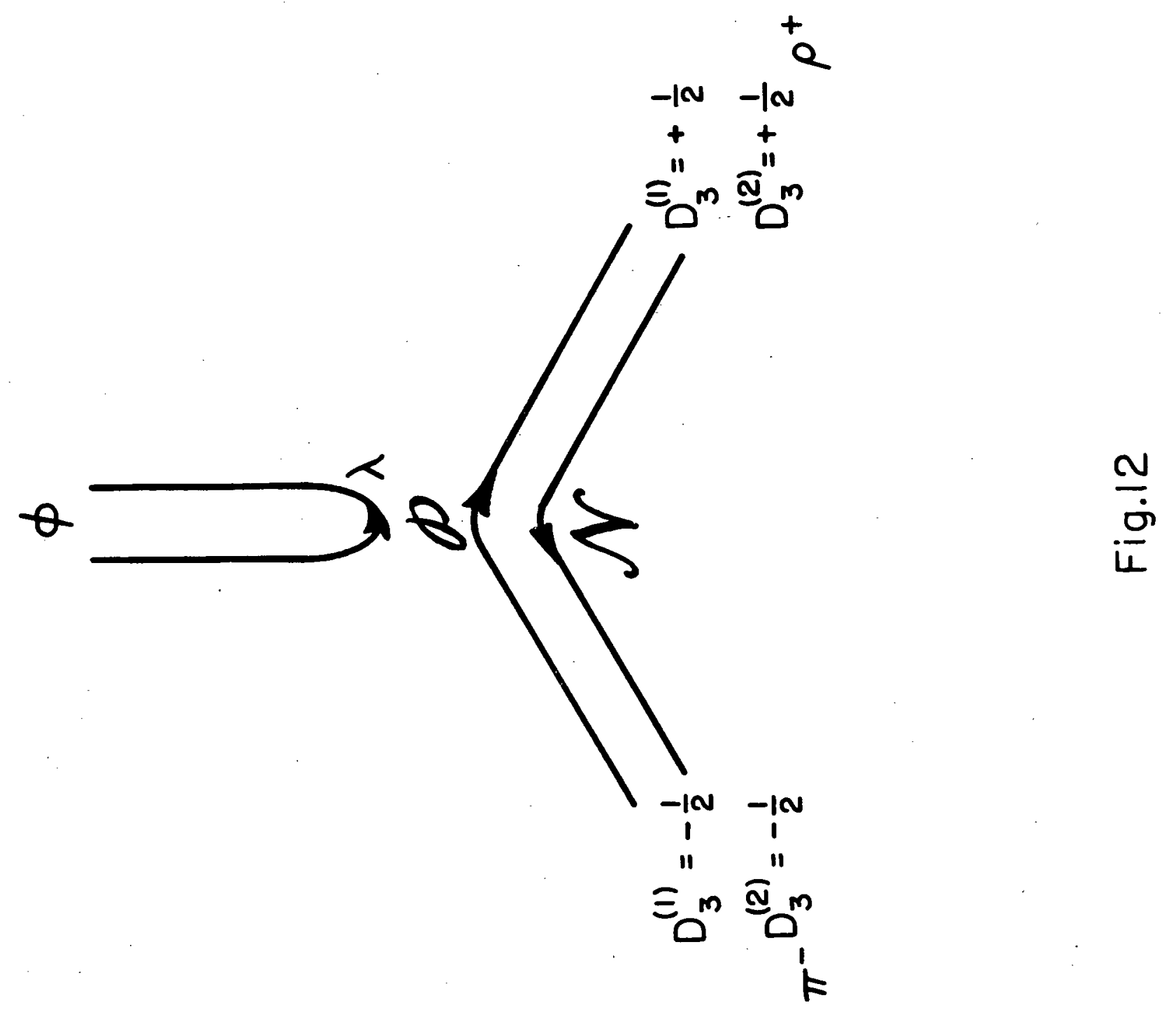

